\title{
On-site Gas Sensing by Surface-Enhanced Raman Scattering
}

\author{
Lingwei $\mathrm{Ma}^{1}$, and Zhengiun Zhang ${ }^{1}$ \\ ${ }^{1}$ Advanced Materials Laboratory, School of Materials Science and Engineering, Tsinghua University, \\ Beijing 100084, China \\ zjzhang@tsinghua.edu.cn
}

\begin{abstract}
:
$\mathrm{Ag}$ nanorods coated with an ultrathin $\mathrm{HfO}_{2}$ shell $\left(\mathrm{AgNRs} @ \mathrm{HfO}_{2}\right)$ were prepared for the synthesis of a versatile, robust, and easily recyclable surface-enhanced Raman scattering (SERS) substrate. This substrate maximizes the high melting point of $\mathrm{HfO}_{2}$ shell and thus ensures the plasmonic efficiency of AgNRs. Therefore, it possesses extraordinary thermal stability and SERS activity, which could act as a reusable and cost-effective SERS detector. After SERS detection, the regeneration of $\mathrm{AgNRs@HfO} 2$ was achieved by annealing the substrate within several seconds. This procedure led to the thermal release of adsorbed molecules and resulted in a refreshed substrate for subsequent measurements. The composite substrate maintained its SERS efficiency well during multiple "detection-heating" cycles, exhibiting good stability and recyclability. Furthermore, in addition to revealing the feasibility of SERS sensing in liquids, AgNRs@ $\mathrm{HfO}_{2}$ also provided continuous real-time monitoring of gas targets at ultralow concentrations. This work provides a robust and renewable SERS sensor with advantages of high sensitivity, stability, cost effectiveness, and easy operation, which can be implemented for both aqueous and gaseous analyte detection and is thus an intriguing candidate for practical applications in environmental, industrial, and homeland security sensing fields.
\end{abstract}

Key words: Surface-enhanced Raman scattering, Ag nanorods, localized surface plasmon resonance, trace level detection, gas sensing.

\section{Introduction}

Surface-enhanced Raman scattering (SERS) is a powerful spectroscopic technique in biomolecule sensing, environmental monitoring, and detection of hazardous substances, etc. Due to localized surface plasmon resonance (LSPR) [1] of metallic nanostructures, SERS offers ultrasensitive and nondestructive sensing of trace analytes. Nevertheless, given that SERS substrates are generally made of noble metals (e.g., $\mathrm{Au}, \mathrm{Ag}$, and $\mathrm{Cu}$ ), the costly preparation and disposable property of metallic substrates seriously obstruct the universality of SERS analysis. In addition, gas sensing is an important part of SERS applications, so it is desired to fabricate sensitive gas sensors.

In this study, a novel AgNRs@ $\mathrm{HfO}_{2}$ SERS platform with $\mathrm{Ag}$ nanorods coated by an ultrathin and uniform $\mathrm{HfO}_{2}$ layer was developed. AgNRs@ $\mathrm{HfO}_{2}$ exhibits superior sensitivity and high-temperature robustness, and could thus function as a reusable and cost-effective SERS detector. After SERS measurement, the regeneration of AgNRs@ $\mathrm{HfO}_{2}$ was achieved by thermal annealing the substrate within several seconds, in which way the adsorbed molecules were removed completely and conveniently. Notably, the composite platform can not only be exploited for SERS determination in aqueous solutions but also shows great potential for in situ gas sensing with ultralow concentrations.

\section{Characterization of AgNRs@HfO}

Slanted AgNRs were prepared by glancing angle deposition (GLAD) technique in an electron-beam system. The $\mathrm{HfO}_{2}$ layers were then coated onto the as-prepared AgNRs via atomic layer deposition (ALD) approach. Figure 1 shows the SEM and TEM images of AgNRs coated with an $\mathrm{HfO}_{2}$ shell of $\sim 1.6 \mathrm{~nm}$. AgNRs were well-separated, and the ultrathin $\mathrm{HfO}_{2}$ shell wrapped the AgNRs uniformly.

\section{Thermal stability of AgNRs@ $\mathrm{HfO}_{2}$}

To achieve a "thermally cleanable" SERS sensor, its high-temperature stability must be assessed first. For bare AgNRs, their structures were damaged at a relatively low temperature of $\sim 100{ }^{\circ} \mathrm{C}$ [2] and, according, they were not 
feasible for high-temperature sensing or thermal cleaning. On the other hand, benefiting from the high melting point of $\mathrm{HfO}_{2}$, the coated substrate maintained well its morphology at temperatures as high as $400{ }^{\circ} \mathrm{C}$. This stable AgNRs@ $\mathrm{HfO}_{2}$ substrate offers us a reliable means to thermally clean it without structure destruction, endowing it with great potential as a renewable SERS detector.
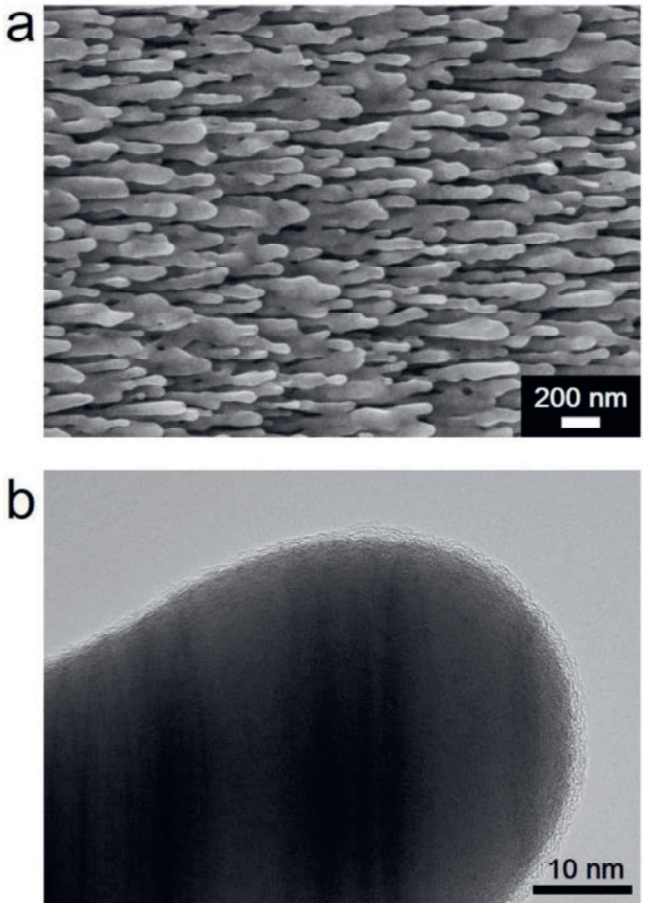

Fig.1. (a) SEM and (b) TEM images of AgNRs@ $\mathrm{HfO}_{2}$ substrates with shell thickness of $\sim 1.6 \mathrm{~nm}$.

\section{Recyclable SERS detection of vapor-phase molecules}

Figure 2a shows the schematic for SERS detection of 2-naphthalenethiol (2-NAT) gas molecules using AgNRs@ $\mathrm{HfO}_{2}$ substrate. $\mathrm{N}_{2}$ was utilized as the carrier gas to transport 2NAT from solution to SERS substrate, and Raman spectra were obtained in situ. Figure $2 \mathrm{~b}$ shows the Raman spectra of 600 ppb 2-NAT and its Raman intensity changes at $1379 \mathrm{~cm}^{-1}$ within $100 \mathrm{~min}$. The SERS signals increased gradually and reached saturation afterwards, demonstrating the feasibility of AgNRs@ $\mathrm{HfO}_{2}$ for trace gas sensing. After Raman detection, the AgNRs@HfO 2 substrate was annealed on a hot plate at $200-300{ }^{\circ} \mathrm{C}$ for a few seconds. Figure $2 \mathrm{c}$ shows the Raman intensity variations of $600 \mathrm{ppb} 2-\mathrm{NAT}$ at $1379 \mathrm{~cm}^{-1}$ peak during the repetition of "vapor exposure-thermal cleaning" cycles on the substrate. AgNRs@HfO exhibited almost no degradation in SERS sensitivity after multiple measurements, and the composite could regenerate rapidly and completely after annealing.
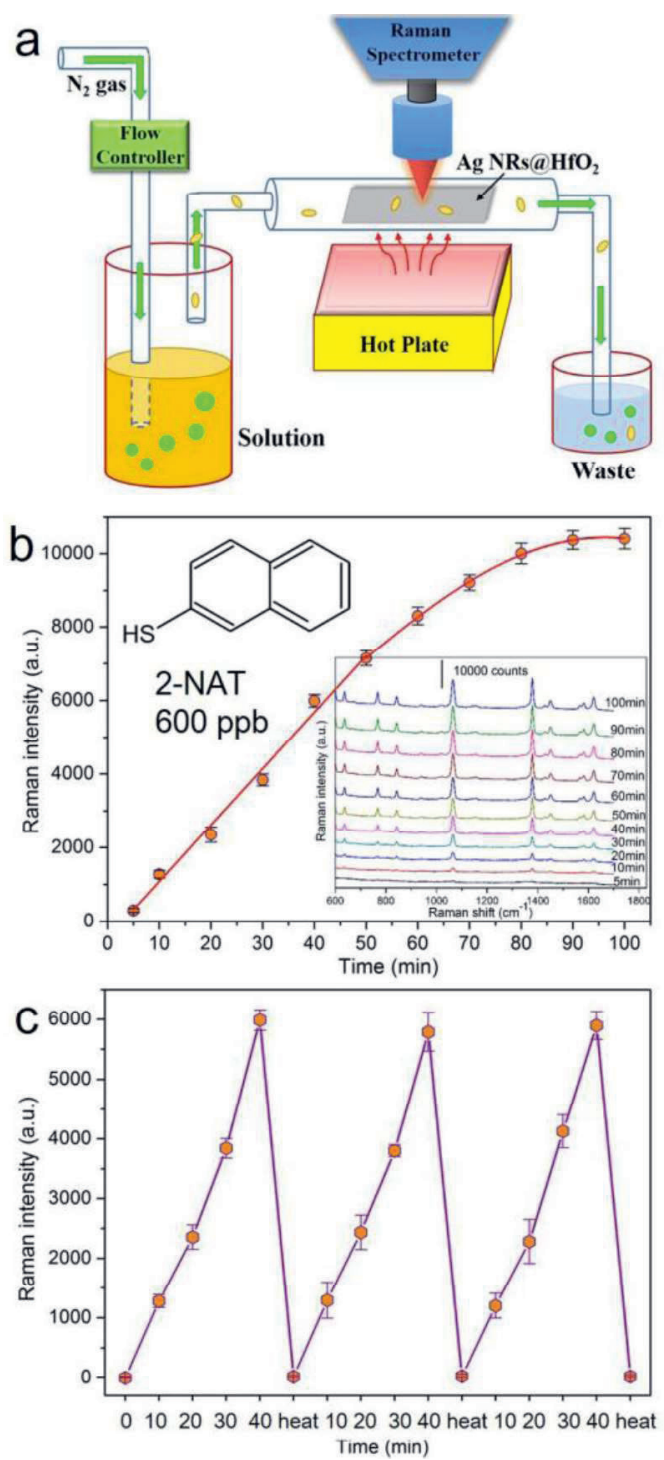

Fig.2. (a) Schematic of the gas sensing device; (b) SERS spectra and the $1379 \mathrm{~cm}^{-1}$ peak intensity of 2NAT on the AgNRs@HfO substrate as a function of gas flow time; (c) $1379 \mathrm{~cm}^{-1}$ peak intensity variations of 2-NAT during the repetition of "vapor exposurethermal cleaning" cycles on the substrate.

\section{Conclusion}

This work leads to the development of efficient, robust and renewable SERS sensors that provide continuous, real-time monitoring of vapor-phase analytes at concentrations down to a ppb molecular level. The AgNRs@ $\mathrm{HfO}_{2}$ substrate provides an exciting platform for rapid and in situ determinations of gas samples from realistic environments, e.g., explosives, volatile organic compounds and chemical warfare agents.

\section{References}

[1] Liz-Marzán, L. M. Langmuir 22, 32-41 (2006); doi: 10.1021/la0513353

[2] Ma L, Huang Y, Hou M, Xie Z, Zhang Z. Scientific Reports 5,12890 (2015); doi: 10.1038/srep12890 